\title{
La transparencia en la administración de los recursos públicos
}

\section{RESUMEN}

\author{
Ronal Atilio Arévalo Montalván \\ paad26@hotmail.com \\ https://orcid.org/0000-0001-8613-0305 \\ Escuela de Posgrado \\ Universidad César Vallejo
}

Hipólito Percy Barbarán Mozo

pbarmozito@hotmail.com

ORCID: 0000-0002-9316-202X

Escuela de Posgrado

Universidad César Vallejo

La corrupción sigue siendo una de las principales preocupaciones de los peruanos sobre la actuación tanto del Estado como de los Gobiernos. Una de las opciones para combatir la corrupción es fomentar cada vez más mecanismos de transparencia en la administración de los recursos públicos. Los funcionarios públicos están llamados a servir a los intereses de la Nación y a ser garantes de la correcta utilización de los recursos públicos de forma transparente. En conclusión el presente artículo presenta orientación cualitativa, de tipo básico de revisión sistémica, "Evidencias disponibles" y está integrado por 09 artículos científicos que son soporte y las bases en donde se solidifican los fundamentos bibliográfico de la misma, para la ejecución del presente estudios; son grandes herramientas de consulta los principales almacenes sistemáticos como el Sistema de Información Científica Redalyc.org, Dialnet, Revistas y Blogs especializados, teniendo en consideración nuestra palabra clave transparencia; administración de recursos, en este conglomerado de análisis en donde existen dos elementos ciudadanía y estado siendo preponderante garantizar la transparencia para una correcta administración de los recursos públicos, después de analizar diversos artículos podemos decir como segunda conclusión que los tres niveles de gobierno pasan por momentos difíciles en lo que refiere a la buena administración de los fondos públicos, que afecta de manera directa al desarrollo de los pueblos y al estado en general, sucede esto debido a la escasa capacitación del personal, autoridades que desconocen aspectos de gestión, poco comunicación con las persona o grupos de personas, la ausencia de planes y políticas públicas bien estructuradas, así como la falta 
de interés de las autoridades, etc., impide que se implementen mecanismos que promuevan el desarrollo integral de los pueblos, La transparencia en la administración de los fondos públicos está estrechamente unida a la calidad democrática. La gestión pública transparente asume el deber de responder a las legítimas demandas sociales, con el compromiso de rendir cuentas y para verificar el cumplimiento de esta misión, es necesario contar con instrumentos que revelen la efectiva comprensión y satisfacción de las necesidades de la población, enmarcado siempre en los principios de honestidad y legalidad. Arévalo, Ronal (2021)

Palabras claves: transparencia; administration de recursos.

Artículo recibido: 18. Junio. 2021 Aceptado para publicación: 26. Julio. 2021

Correspondencia:paad_26@hotmail.com Conflictos de Interés: Ninguna que declarar 


\title{
Transparency in the administration of public resources
}

\begin{abstract}
Corruption continues to be one of the main concerns of Peruvians regarding the performance of both the State and the Governments. One of the options to combat corruption is to promote more and more transparency mechanisms in the administration of public resources. Public officials are called to serve the interests of the Nation and to be guarantors of the correct use of public resources in a transparent manner. In conclusion, this article presents a qualitative orientation, a basic type of systemic review, "Available evidence" and is made up of 09 scientific articles that are support and the bases on which the bibliographic foundations of the same are solidified, for the execution of the present studies; The main systematic warehouses such as the Redalyc.org Scientific Information System, Dialnet, Magazines and specialized Blogs are great consultation tools, taking into consideration our keyword transparency; administration of resources, in this conglomerate of analysis where there are two elements citizenship and state, being preponderant to guarantee transparency for a correct administration of public resources, after analyzing various articles we can say as a second conclusion that the three levels of government go through moments difficult in terms of the good administration of public funds, which directly affects the development of the towns and the state in general, this happens due to the scarce training of the personnel, authorities that are unaware of management aspects, little communication with the person or groups of people, the absence of well-structured public plans and policies, as well as the lack of interest of the authorities, etc., prevents the implementation of mechanisms that promote the integral development of the towns, Transparency in the administration of public funds is closely linked to democratic quality. Transparent public management assumes the duty to respond to legitimate social demands, with the commitment to be accountable and to verify compliance with this mission, it is necessary to have instruments that reveal the effective understanding and satisfaction of the needs of the population, framed always in the principles of honesty and legality. Arevalo, Ronal (2020)
\end{abstract}

Key words: transparency; resource management. 


\section{INTRODUCCIÓN}

En el presente artículo el concepto de "transparencia" alude a la obligación de los poderes públicos de rendir cuentas de sus acciones, es decir, a exponer y someter al juicio de la población la información relativa a su administración, a las bases de formulación de sus decisiones, al destino de los recursos que la sociedad les ha confiado y al desempeño de sus agentes. Arévalo Ronal (2020). La corrupción genera, entre otros efectos, la deslegitimación del Estado, y de la organización política, afecta la eficacia empresarial, hace ineficiente la asignación de recursos públicos y privados y distorsiona las políticas públicas. Bejarano, Jesús Antonio (1996). Es aquí donde la democracia y la transparencia deben hacer su trabajo y entre ellos existe una estrecha vinculación, ya que el acceso a la información y otros aspectos elevan la exigencia sobre la acción pública y a la vez, hace que los ciudadanos tomen decisiones mejor fundamentadas en el curso de los procesos democráticos. La transparencia es una de las variables de la ecuación de la gobernabilidad democrática (Peschard, J. (2008)

Dentro de la creciente incapacidad de nuestro sistema político está la desigualdad social vinculada a las causas de la corrupción, entonces ¿a más desigualdad social mayor corrupción?, como, por ejemplo. Mala distribución de la política del poder en la administración pública, inexistente ejercicio transparente de información, falta de conciencia social, desconocimiento de lo legal e ilegal, falta de valores, ética y moral, impunidad en los actos de corrupción, poca eficiencia de la administración pública, excesivo poder discrecional del funcionario público, ambición, codicia. Estas causas nos conllevan a consecuencia Políticas, perjudica el funcionamiento de las instituciones públicas, afecta la eficacia y la eficiencia en las instituciones públicas, afecta a la democracia y la gobernabilidad de los pueblos, consecuencias económicas, inestabilidad política de los Estados, genera menos inversionista, consecuencia social, pobreza, pérdida de credibilidad en las instituciones públicas, incremento de la delincuencia por pérdida de oportunidades, la corrupción mina el desarrollo y progreso de los pueblos. Estudios realizados por el INEI, el 75\% de la economía peruana es informal. Sin embargo, se sabe poco sobre la relación entre corrupción e informalidad en el país. Existiendo una asociación positiva entre corrupción y desigualdad, es decir, la corrupción incrementa el nivel de empleo en el sector informal. Ludwig Huber (2017). Ante estas acciones de deterioro de las políticas públicas y por ende de la 
administración de nuestros recursos públicos presentamos herramientas orientadas a corregir estos resultados que dañan nuestra visión de país. Los objetivos definidos del presente artículo es proporcionar a las entidades públicas, herramientas solidas como la cultura de la rendición de cuentas, que es, simplemente, el acto en el que los funcionarios y gobernantes le muestran a los ciudadanos los avances de los proyectos, las dificultades de la ejecución y los resultados de su gestión. Estos engranajes de desarrollo se deben instaurar en nuestra política peruana, siendo cinco pilares y tres ejes transversales que coadyuvan a la gestión integral de los mismos. Los pilares son el alineamiento de las políticas públicas, los planes Estratégicos y operativos, los presupuesto por objetivos, gestión por procesos, (simplificación administrativa y organización institucional) servicio civil meritocratico y el seguimiento, evaluación y gestión del conocimiento. Y los tres ejes transversales son: Gobierno abierto, gobierno electrónico y, articulación intersectorial.

El desempeño de las instituciones públicas en la sociedad contemporánea, es una tarea que exige responsabilidad, eficiencia, eficacia, calidad y transparencia, dado que esas atribuciones permiten dar cumplimiento a una diversidad de objetivos, metas y políticas públicas que están orientadas a la comunidad para preservar el bien común. En esto cabe destacar las competencias otorgadas por cada gobierno a la Contraloría General de la República de Perú, principalmente aquellas relacionadas con el control a la gestión en el desempeño institucional de la administración pública, señalando con énfasis que es un sistema dinámico e importante para el logro de metas organizacionales representado por la eficiencia, eficacia, calidad y transparencia con que se desarrollan las actividades que permitan lograr los objetivos y metas que busca satisfacer a una población Yokir Reyna Zambrano (2017)

\section{MÉTODO.}

En el artículo se ha efectuado el método de recopilación de información por medio de la revisión sistematizada de artículos científicos, revistas, tesis, y blogs en la webb, utilizando como palabras clave “Transparencia; administración de recursos".se identificaron, analizaron e interpretaron diversos aspectos en los cuales se desarrollan actividades de gestión pública, dándome la oportunidad de inyectar mi conocimiento, experiencia académica y laboral con la finalidad de emitir precisiones acertadas que enriquezcan la presente investigación. La investigación fue básica de diseño descriptivo, 
debido a que se ha detallado el comportamiento de las variables en una efectividad determinada, buscando a su vez proponer medidas o estrategias que ayuden a solucionar las imperfecciones presentadas en el contexto de la investigación mediante la formulación de medidas correctivas. Mario Tamayo y Tamayo. (2006).

Es menester recalcar que estos tipos de estudios son primarios y constituyen una herramienta esencial para sintetizar la información científica disponible, incrementar la validez de las conclusiones de estudios individuales e identificar áreas de incertidumbre donde sea necesario realizar investigación. Hernández, Sampieri, (2016).

Para la ejecución del presente estudio la herramienta utilizada fue tabla de registro estructurado en relación a las principales cualidades o características de los artículos consultados, considerando para ello el autor, año, tipo y diseño y conclusiones respectivamente; donde los criterios permitirán analizar la información presentada como resultados. Naupas, Valdivia, Palacios y Romero, (2018). Finalmente, se respetaron los lineamientos estipulados en las Normas APA $7^{\text {a }}$ edición, para que las citaciones de los diversos autores de consulta que publican y exponen temas relacionados con la investigación, se realicen de manera correcta, teniendo en consideración el espacio y tiempo los derechos de autor, es propicio dar a conocer que se respetó los conductos o pautas establecidas por la Universidad César Vallejo, en el proceso de desarrollo del presente estudio.

\section{RESULTADOS.}

Mediante la revisión efectuada, se procede a presentar la siguiente tabla como parte de los resultados:

\begin{tabular}{|c|c|c|c|c|l|}
\hline $\mathbf{N}^{\circ}$ & \multicolumn{1}{|c|}{ Autor } & País & Clasificación & Tipo y Diseño & \multicolumn{1}{|c|}{ Conclusión } \\
\hline 1 & $\begin{array}{l}\text { Bejarano, Jesús Antonio } \\
(1996)\end{array}$ & Colombia & $\begin{array}{c}\text { Artículo } \\
\text { Científico }\end{array}$ & $\begin{array}{l}\text { No experimental } \\
\text { descriptivo }\end{array}$ & $\begin{array}{l}\text { La corrupción genera, entre otros } \\
\text { efectos, la deslegitimación del Estado, } \\
\text { y de la organización política, afecta la } \\
\text { eficacia empresarial, hace ineficiente la } \\
\text { asignación de recursos públicos y } \\
\text { privados y distorsiona las políticas } \\
\text { públicas. }\end{array}$ \\
\hline 2 & Peschard, J. (2008). & México & $\begin{array}{l}\text { Artículo } \\
\text { Científico }\end{array}$ & $\begin{array}{l}\text { Na democracia y la transparencia } \\
\text { tienen una estrecha vinculación, ya que } \\
\text { el acceso a la información eleva la } \\
\text { exigencia sobre la acción pública y a la } \\
\text { vez, hace que los ciudadanos tomen } \\
\text { decisiones mejor fundamentadas en el } \\
\text { curso de los procesos democráticos. La } \\
\text { transparencia es una de las variables de } \\
\text { la ecuación de la gobernabilidad } \\
\text { democrática. }\end{array}$ \\
\hline
\end{tabular}




\begin{tabular}{|c|c|c|c|c|c|}
\hline 3 & Ludwig Huber (2017) & Perú & $\begin{array}{l}\text { Artículo } \\
\text { Científico }\end{array}$ & $\begin{array}{l}\text { No experimental } \\
\text { descriptivo }\end{array}$ & $\begin{array}{l}\text { La corrupción mina el desarrollo y } \\
\text { progreso de los pueblos. Estudios } \\
\text { realizados por el INEI, el } 75 \% \text { de la } \\
\text { economía peruana es informal. Sin } \\
\text { embargo, se sabe poco sobre la } \\
\text { relación entre corrupción e } \\
\text { informalidad en el país. } \\
\text { Existe asociación positiva entre } \\
\text { corrupción y desigualdad, es decir, la } \\
\text { corrupción incrementa el nivel de } \\
\text { empleo en el sector informal. }\end{array}$ \\
\hline 4 & $\begin{array}{l}\text { Yokir Reyna Zambrano } \\
\text { (2017) }\end{array}$ & Ecuador & $\begin{array}{l}\text { Artículo } \\
\text { Científico }\end{array}$ & $\begin{array}{l}\text { No experimental } \\
\text { descriptivo }\end{array}$ & $\begin{array}{l}\text { cabe destacar las competencias } \\
\text { otorgadas por cada gobierno a la } \\
\text { Contraloría General de la República, } \\
\text { principalmente aquellas relacionadas } \\
\text { con el control a la gestión en el } \\
\text { desempeño institucional de la } \\
\text { administración pública, señalando con } \\
\text { énfasis que es un sistema dinámico e } \\
\text { importante para el logro de metas } \\
\text { organizacionales representado por la } \\
\text { eficiencia, eficacia, calidad y } \\
\text { transparencia con que se desarrollan } \\
\text { las actividades que permitan lograr los } \\
\text { objetivos y metas que busca satisfacer } \\
\text { a una población }\end{array}$ \\
\hline 5 & $\begin{array}{l}\text { Mario Tamayo y Tamayo } \\
\text { ( 2006) }\end{array}$ & Colombia & $\begin{array}{l}\text { Artículo } \\
\text { Científico }\end{array}$ & $\begin{array}{l}\text { No experimental } \\
\text { descriptivo }\end{array}$ & $\begin{array}{l}\text { La investigación fue básica de diseño } \\
\text { descriptivo, debido a que se ha } \\
\text { detallado el comportamiento de las } \\
\text { variables en una efectividad } \\
\text { determinada, buscando a su vez } \\
\text { proponer medidas o estrategias que } \\
\text { ayuden a solucionar las imperfecciones } \\
\text { presentadas en el contexto de la } \\
\text { investigación mediante la formulación } \\
\text { de medidas correctivas }\end{array}$ \\
\hline 6 & $\begin{array}{l}\text { Hernández Sampieri, } \\
\text { (2016.) }\end{array}$ & México & $\begin{array}{l}\text { Artículo } \\
\text { Científico }\end{array}$ & $\begin{array}{l}\text { No experimental } \\
\text { descriptivo }\end{array}$ & $\begin{array}{l}\text { estos tipos de estudios son primarios y } \\
\text { constituyen una herramienta esencial } \\
\text { para sintetizar la información científica } \\
\text { disponible, incrementar la validez de } \\
\text { las conclusiones de estudios } \\
\text { individuales e identificar áreas de } \\
\text { incertidumbre donde sea necesario } \\
\text { realizar investigación }\end{array}$ \\
\hline
\end{tabular}




\begin{tabular}{|c|c|c|c|c|c|}
\hline 7 & $\begin{array}{l}\text { Gómez González, Arely } \\
\text { (2018) }\end{array}$ & México & $\begin{array}{l}\text { Artículo } \\
\text { Científico }\end{array}$ & $\begin{array}{l}\text { No experimental } \\
\text { descriptivo }\end{array}$ & $\begin{array}{l}\text { Tengamos en consideración que un } \\
\text { buen gobierno no solo requiere } \\
\text { funcionarios responsables sino también } \\
\text { políticos responsables, puesto que son } \\
\text { éstos últimos principalmente quienes } \\
\text { gozan del máximo margen de } \\
\text { autonomía en las decisiones y de estas } \\
\text { decisiones depende a su vez la } \\
\text { actuación de los principios; y por otra } \\
\text { parte sobre la Mejora de la gestión } \\
\text { pública es a base de la eficiencia } \\
\text { gubernamental y prevención de la } \\
\text { corrupción }\end{array}$ \\
\hline 8 & $\begin{array}{l}\text { Aprobada por la X } \\
\text { Conferencia } \\
\text { Iberoamericana de } \\
\text { Ministros de } \\
\text { Administración Pública y } \\
\text { Reforma del Estado San } \\
\text { Salvador, El Salvador, } 26 \\
\text { y } 27 \text { de junio de(2008) }\end{array}$ & $\begin{array}{l}\text { San } \\
\text { Salvador. }\end{array}$ & $\begin{array}{l}\text { Resolución } \mathrm{N}^{\circ} \\
\text { 25. Plan de } \\
\text { acción- San } \\
\text { Salvador. } \\
\text { Cumbre } \\
\text { Iberoamericana } \\
\text { de Jefes de } \\
\text { Estado y de } \\
\text { Gobierno }\end{array}$ & $\begin{array}{c}\text { No experimental } \\
\text { descriptivo }\end{array}$ & $\begin{array}{l}\text { Con la finalidad de mejorar la calidad } \\
\text { en los servicios públicos, el Perú } \\
\text { suscribió en el año 2008 la Carta } \\
\text { Iberoamericana de Calidad en Gestión } \\
\text { Pública, que tiene por propósito } \\
\text { promover el establecimiento de un } \\
\text { enfoque común en la formulación de } \\
\text { políticas, planes, modelos y } \\
\text { mecanismos que permitan a las } \\
\text { entidades Públicas la mejora continua } \\
\text { en la calidad de sus servicios }\end{array}$ \\
\hline 9 & $\begin{array}{l}\text { Saravia Salazar J.I. } \\
\text { (2018). }\end{array}$ & Perú & $\begin{array}{l}\text { Artículo } \\
\text { Científico }\end{array}$ & $\begin{array}{c}\text { No experimental } \\
\text { descriptivo }\end{array}$ & $\begin{array}{l}\text { Uno de los principales inconvenientes } \\
\text { para la consolidación de una adecuada } \\
\text { gestión pública en nuestro país ha sido } \\
\text { y es la poca capacidad de gestión y } \\
\text { preparación de los funcionarios } \\
\text { públicos al momento de asumir los } \\
\text { retos que la sociedad y la } \\
\text { administración les exige. }\end{array}$ \\
\hline
\end{tabular}

La información y el título que se presenta tiene prevalencia de 3 artículos de autores mexicanos, 2 de Colombia, 2 de Perú, 1 de Ecuador y 1 del salvador, cuyos artículos integran temas de transparencia en la administración de los recursos públicos, e investigación. Es verdad que en la actualidad el estado ha sido uno de los objetos de estudio más importantes en las ciencias sociales, ha sido cuestionado, deslegitimado, demonizado y santificado desde diversidad de enfoques e ideologías. Con la revisión de las referencias bibliográficas relacionadas con la transparencia y la administración de los recursos públicos se ha podido reconocer que los organismos públicos de nuestro país se preocupan por utilizar las mejores herramientas y estrategias establecidos por ley para mejorar sus ejecuciones presupuestales, y que estas ejecuciones del presupuesto se oriente a los objetivos definidos, y que contribuyan con la efectividad de la transparencia y la administración de los recursos públicos. Tengamos en consideración 
que un buen gobierno no solo requiere funcionarios responsables sino también políticos responsables, puesto que son éstos últimos principalmente quienes gozan del máximo margen de autonomía en las decisiones y de estas decisiones depende a su vez la actuación de los principios; y por otra parte la mejora de la gestión pública tiene su base en la eficiencia gubernamental y prevención de la corrupción Gómez González, (2018), de ahí que la mejora de la administración de los recursos públicos implica un mejor aprovechamiento de dichos procesos para maximizar la calidad de los trámites y servicios que presta la Administración de los fondos. Las causas de la mala administración de los recursos es que la población no percibe la mejora, el desarrollo social, económico y la calidad de servicios en las diferentes entidades de gobierno público, la sociedad sigue enfrentándose a la baja valoración de nuestras autoridades nacionales, regionales y locales a lo largo del tiempo, por todos estas debilidades se ha implementado un sistema normativo que viene regulando la conducta ética de los servidores públicos como es la Ley del Código de Ética de la Función Pública Ley No 27815. Promulgada el año (2002) con la finalidad de que la función pública esté al servicio a la nación y con obtención de mayores niveles de eficiencia del aparato estatal para que se logre una mejor atención a la ciudadanía, priorizando y optimizando el uso de los recursos públicos conforme a lo dispuesto por la Ley Marco de Modernización de la Gestión del Estado, Es así que, dentro del contexto internacional, se pudo identificar que los principales debilidades que presentan los organismos del estado durante la ejecución de su presupuesto son: La falta de efectividad de planes y políticas públicas, autoridades sin conocimiento de la normativa de gestión, funcionarios y trabajadores con escasa capacidad en TICs y administración, uniéndose a ello un sistema de planeamiento desconectado, quiere decir que el estado no necesariamente recoge las necesidades de la población y por ende realiza planes que no están articulados con las brechas que debe cubrir, por lo tanto el planeamiento no termina siendo una herramienta efectiva de gestión y no se alinea con el presupuesto público, dentro estas debilidades esta una estructura de organización y funciones ineficaces., las organizaciones de decenas de entidades públicas en américa latina no están diseñadas de acuerdo a sus objetivos, pueden ser porque fueron diseñados con una organización jerárquica sin claridad en los procesos que realizan para entregar sus servicios de manera oportuna y responsable, presentan producción de bienes y servicios inadecuados la mayoría de 
instituciones no tienen los recursos ni capacidades para optimizar sus procesos, por otra parte uno de los problemas más importantes es la desarticulación entre los sistemas administrativos, por ello solo le interesa cumplir sus funciones sin buscar la satisfacción de la ciudadanía, asociado a ello una articulación gubernamental débil, los mecanismos de coordinación establecidos en nuestro marco legal suelen contar con limitaciones en su diseño y han sido poco efectivos al momento de la articulación entre instituciones. Es por ello que, a través de los años, la presencia de la corrupción en los organismos estatales ha sido criticada y enfrentada, pero, lastimosamente, los cambios institucionales que ha sufrido el Estado en su constante diversificación de roles no ha venido acompañada del fortalecimiento de las instituciones fiscalizadoras, a las que no se les ha brindado las atribuciones, ni la autonomía ni la continuidad suficientes para hacerle mella. Los recientes acontecimientos en nuestro país muestran vivamente como la incompetencia y la corrupción pueden causar más destrucción que los fenómenos naturales. Saravia Salazar J.I. (2018). Este debe ser mejorado para que la descentralización nacional pueda ser una realidad.

\section{DISCUSIÓN.}

El presente artículo planteó como objetivo principal Analizar la relación entre la transparencia y la administración de los recursos públicos que caracterizan a las instituciones de gobierno local, Partiendo de las primicias de transparencia en la administración de los recursos públicos y estrategias de gestión, en cuanto al tipo y diseño de investigación, se reconocen que la mayoría de los artículos emplearon diseños no experimentales, a nivel descriptivo, documental. Grupos que se componen de un conjunto de juicios y de reglas que sirve para orientar razonablemente el cumplimiento de los planes de inversión anual de los organismos públicos de nuestro país, estas reglas deben conjugar con el comportamiento funcional de los servidores públicos; los ciudadanos nos preguntamos, de qué manera pre vendríamos la corrupción en los organismos públicos en nuestro país? La prevención no va por la creación de leyes, ya que éstas no son políticas de Estado, sino debemos de buscar cambio en la política educativa, reforzar los valores a nivel institucional y social, la concientización a los buenos modales en la administración de los recursos del estado deberá iniciar en la educación básica primaria, como política gubernamental, es la solución a largo plazo, con esta política de estado se corregirá todo acto doloso, reñido por nuestra sociedad y 
que hace gran daño a la economía nacional, atentando en gran escala a la inversión pública,

El 70\% de los peruanos considera que la corrupción ha sido el principal problema del país durante los últimos 50 años. Lo precisa la Encuesta Nacional de Valores y Ciudadanía (2020), elaborada por el Proyecto Especial Bicentenario de la Presidencia del Consejo de ministros (PCM). La atención eficiente y satisfacción de los ciudadanos es uno de los objetivos fundamentales de las Administraciones Públicas en todo el mundo. El impacto de las tecnologías de la información junto con el mayor nivel de transparencia exigida por la ciudadanía genera ciudadanos más informados y exigentes con la calidad de los servicios que esperan de la Administración. La calidad en los servicios públicos se define, en la Carta Iberoamericana de Calidad en Gestión Pública (2008), como el conjunto de los métodos y estrategias de trabajo que aseguren que dichos servicios cuenten con especificaciones que satisfagan las necesidades y requerimientos de los ciudadanos.

Con la finalidad de mejorar la calidad en los servicios públicos, el Perú suscribió en el año (2008) la carta iberoamericana de calidad en gestión pública, que tiene por propósito promover el establecimiento de un enfoque común en la formulación de políticas, planes, modelos y mecanismos que permitan a las entidades Públicas la mejora continua en la calidad de sus servicios. Aprobada por la X Conferencia Iberoamericana de Ministros de Administración Pública y Reforma del Estado San Salvador, fruto de este compromiso se han ido desarrollando acciones normativas en diferentes administraciones del estado peruano, enfocadas a la mejora, transparencia y eficiencia en la calidad de los servicios prestados a la ciudadanía, poco se ha avanzado es por ello que en líneas arriba se propone la creación de una política pública que inicie en educación básica "primaria" el conocimiento de la estructura del estado, el respeto y a la correcta administración de nuestros recursos, de lo contrario seguiremos sumidos en actos que cada día minan nuestra convaleciente economía y moral de nuestro país.

\section{CONCLUSIONES.}

Las principales debilidades que viven nuestras instituciones públicas en Perú y américa latina sobre su gestión, administración y ejecución de proyectos es similar en estos países de la región, se evidencia la presencia de diversas deficiencias e inconvenientes vinculados a las políticas públicas y otros aspectos que los vincula, dentro de las cuales 
destaca la ausencia de planes y políticas claras y efectivas, falta de conocimientos y preparación de las autoridades, servidores públicos locales con escaso conocimiento de TICs y administración, falta de acciones y medidas estratégicas que permitan asegurar el cuidado y reducción del daño a la economía nacional, regional y local. Ante la adversidad nuestros países de la región luchan por lograr su modernización, para lograr la ansiada modernidad hemos empezado a evaluar nuestra Administración Pública con el fin de incorporar prácticas que garanticen una gestión más efectiva. En este sentido se recomienda implementar herramientas de gestión de la calidad que resulten útiles para identificar las prácticas que harán que la gestión del estado camine hacia la excelencia. Estas son las cinco mejores herramientas: Fomentar el liderazgo, que es una de las piedras angulares de los modelos de excelencia. Dirigir una organización sin perder de vista la misión, los valores y los objetivos, permite incrementar el rendimiento de los equipos, motivar a las personas y convertirse en un modelo a seguir. Por su puesto Haciendo uso de la estrategia, es decir sabiendo hacia dónde vamos, cuál es el objetivo y cómo vamos a lograrlo puede marcar la diferencia entre una gestión exitosa y una que no lo sea. La estrategia, además, requiere contar con información precisa sobre los asuntos públicos que se van a resolver. Gestionar efectivamente los recursos humanos, todos conocemos que el manejo de los equipos dentro de las instituciones es clave para garantizar gestiones públicas más efectivas. Desde la selección adecuada del personal hasta su ubicación estratégica en cargos acordes a sus capacidades. "Meritocracia". La gestión para resultados, debemos tener en claro lo que se quiere alcanzar, cuál es el objetivo o la meta a alcanzar en bienes, servicios u obras públicas, es vital en la administración gubernamental, para alinear las diferentes y diversas actividades que se desarrollan en el estado, y por último mejorar los procesos, ninguna gestión pública podrá alcanzar el éxito sin procesos que funcionen adecuadamente. Si los procesos no funcionan, se pierde todo el objetivo de la gestión que es servir a las personas. Todas estas herramientas enmarcadas en la información pública, en aras de la trasparencia. Arévalo, Atilio (2020)

\section{REFERENCIAS BIBLIOGRÁFICAS.}

Álvaro Ramírez A. Daniela Rosales A. (2017). Desde el gobierno abierto al Estado abierto en América Latina y el Caribe. Comisión Económica para América Latina y el Caribe (CEPAL) Santiago de chile 
Bejarano, Jesús A. (1996) Estrategias contra la corrupción. En: Descentralización y corrupción. Bogotá Fescol (Ed).

Carta Iberoamericana de Calidad en Gestión Pública (2008). Aprobada por la X Conferencia Iberoamericana de Ministros de Administración Pública y Reforma del Estado San Salvador, El Salvador, 26 y 27 de junio de 2008. Adoptada por la XVIII Cumbre Iberoamericana de Jefes de Estado y de Gobierno San Salvador, El Salvador, del 29 al 31 de octubre de 2008.

Contraloría General de la República. (2014). Estudio del proceso de descentralización en el Perú. Estudio auspiciado por: Programa de las Naciones Unidas para el Desarrollo PNUD

Gómez González A, 2018. Seminario RRC. www.rendiciondecuentas.org.mx

Ludwing Huber A. (2016). Corrupción y transparencia” (Instituto de Estudios Peruanos IEP), incluye el libro "Balance de Investigación en Políticas Públicas 20112016. Consorcio de Investigación Económico y Social "CIES"

Martínez Cárdenas E, y Ramírez Mora J. (2010). La corrupción en la administración pública: un perverso legado colonial con doscientos años de vida republicana, Universidad Nacional de Bucaramanga (Ed)

Martín, R. U, (2019), Incidencias de la Tecnología web 2.0 en el contexto de la gobernanza y la gobernabilidad, Universidad Nacional Experimental Francisco de Miranda (Venezuela).

Peschard, Jacqueline (2008). Transparencia y Partidos Políticos. Cuaderno de Transparencia, $\mathrm{N}^{\mathrm{o}}$ 8. México: Instituto Federal de Acceso a la Información Pública (IFAI).

Reyna Zambrano M. Y. (2017). El control a la gestión en la administración pública: una mirada a las legislaciones de Ecuador y Perú. Universidad San Gregorio de Portoviejo. Ecuador, myreyna@sangregorio.edu.ec, artículo publicado: 31 de diciembre de 2017.

Saravia Salazar J.I. (2018). La gestión pública en el Perú en perspectiva histórica (siglos XIX-XXI). Universidad Nacional Mayor de San Marcos

SIMÓN AYLAS, W. A, (2016), Gobierno Electrónico y su Influencia en la Gestión Pública de la Municipalidad Distrital de Yanacancha Pasco - Universidad Nacional Daniel Alcides Carrión, (Pasco) 
Torres Manrique, J.I, (2013). Una mirada crítica a los derechos a la transparencia, acceso a la información pública y rendición de cuentas. publicación: 01/10/2013. Abogado por la Universidad Católica de Santa María (Arequipa - Perú). 